\title{
Method of the Year 2012
}

\author{
New method and tool developments are helping to bring targeted proteome analysis \\ technologies to a broader array of biologists.
}

At the close of 2012 at Nature Methods, we continue our annual celebration of biological research methods with our choice of targeted proteomics as Method of the Year.

Our choice is a little different from those of previous years, when we highlighted very new, cutting-edge techniques such as next-generation sequencing (2007), super-resolution microscopy (2008) and optogenetics (2010). Broadly speaking, methods for targeted protein analysis date back to the introduction of the radioimmunoassay in the 1960s. Antibody reagents have long been indispensable research tools, allowing biologists to pull out their protein of interest from a complex biological sample or detect their protein in a tissue slice or in a western blot.

In modern research applications such as immunofluorescence microscopy, flow cytometry and protein microarray technology, antibodies continue to be vitally important. But reliance on antibodies for protein detection has drawbacks. The biggest limitations are that antibodies vary greatly in availability and quality. Large-scale efforts such as the Human Protein Atlas, Antibodypedia and the US National Institutes of Health Protein Capture Reagents Program, among others, seek to address these limitations through the systematic generation and characterization of antibodies with respect to human protein targets.

But there is another way. Mass spectrometry, perhaps most familiar for its use in discovery-based proteomics, can also be applied to specifically analyze target proteins of interest. In the most mature technology for targeted analysis, known as selected (or multiple) reaction monitoring, a mass spectrometer called a triple quadrupole is programmed to detect specific peptides that uniquely represent proteins of interest, allowing researchers to quantitatively monitor these proteins with high sensitivity and reproducibility. A Primer on page 23 provides a brief overview of this technology and compares and contrasts the targeted mass spectrometry workflow with discovery-based proteomics analysis.

Mass spectrometry has an advantage over antibodies in that developing a new targeted assay is much faster than generating a new antibody, and issues of detection specificity (that is, antibody cross-reactivity) are greatly minimized. Antibodies still have the upper hand in terms of sensitivity of detection for low-abundance proteins. But a highly positive trait of mass spectrometry is its inherent ability to unambiguously detect multiple proteins in one experiment, allowing, for example, a systems biology researcher to look at what happens to protein levels upon perturbation of a protein network or a clinical researcher to measure how a panel of proposed biomarkers changes in a disease state.

Getting to the current state of affairs in the mass spectrometry-based targeted proteomics field has taken considerable time. The triple quadrupole mass spectrometer was developed in the 1970s, and the first demonstrations that it could be used to detect peptides were published in the 1980s. Interest in applying targeted mass spectrometry technology on a broader scale has been rapidly escalating in the past several years, and new methods, tools, resources and next-generation approaches are helping to bring the technology to a wider community of researchers. In a News Feature on page 19, Vivien Marx describes how mass spectrometry-based targeted proteomics technologies are on the cusp of translation to biological laboratories.

Mass spectrometry-based proteomics has long been viewed as far too complex for anyone but specialists to apply. A discovery-based proteomics experiment requires highly sophisticated bioinformatics aptitude to extract reliable results from the data. On the other hand, targeted mass spectrometry experiments are in principle simple to perform, once reliable protein assays are available, and data analysis is relatively straightforward. Targeted approaches also allow biologists to carry out fundamentally different kinds of experiments-driven by hypotheses-in contrast to discovery-based investigations. In a Commentary on page 24, Ruedi Aebersold, Paola Picotti and Bernd Bodenmiller make a strong case for the important role that mass spectrometry can play in hypothesis-driven research, perhaps even eventually replacing the tedious western blot in basic research labs.

Though Nature Methods' focus is on methods for basic biological research, we cannot ignore the important impact that targeted mass spectrometry methods are having on the field of clinical proteomics. In a Perspective on page 28, Steven Carr and Michael Gillette discuss how selected reaction monitoring is being used to sensitively detect proteins in plasma and tissue and how it is highly suited for verification of human disease biomarker candidates.

And finally, as every year, we look to the future with our selection of Methods to Watch (starting on page 35), presenting a mixture of diverse technologies that we are keeping our eyes on: some poised to make an impact in the very near future, others in the longer term.

We hope you enjoy our special feature. To all readers, a happy new year! 